\title{
An Oral Abscess as the Entrance Leading to Endocarditis: A Sexual Behavior Role
}

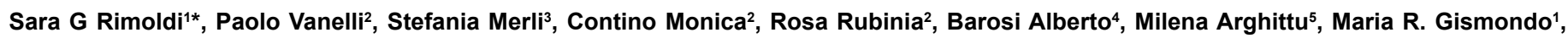
Giovanni Cagnoni ${ }^{2}$ and Carlo Antona ${ }^{2}$

${ }^{1}$ Laboratory of Clinical Microbiology, Virology and Diagnostics Bioemergenze, ASST Fatebenefratelli Sacco-Polo Universitario, Via G.B. Grassi 74, 20157 Milan, Italy

${ }^{2}$ Cardiac Surgery Unit, ASST Fatebenefratelli Sacco- Polo Universitario, Via G.B. Grassi 74, 20157 Milan, Italy

${ }^{3}$ Division of Infectious Diseases, Azienda Ospedaliera-Polo Universitario Luigi Sacco, Via G.B. Grassi 74, 20157 Milano, Italy

${ }^{4}$ U.O. Cardiology, ASST Fatebenefratelli Sacco- Polo Universitario, Via G.B. Grassi 74, 20157 Milan, Italy

${ }^{5}$ Laboratory of Microbiology, IRCCS Policlinico-Milano, Via F.Sforza 20155, Milan, Italy

\begin{abstract}
We report a case of atypical endocarditis for which the transmission mode has long been debated among cardiac surgeons, microbiologists, and infectious disease specialists. This is the first reported case of Streptococcus agalactiae being responsible for the probable sexually transmitted case of endocarditis in a healthy, 49-year-old female.
\end{abstract}

Keywords: S. agalactiae; Infective endocarditis; Aortic valve

\section{Introduction}

The risk factors for infective endocarditis (IE) in the population are changing [1]: the reported worldwide increase in the incidence of IE has been associated with the widespread use of medical devices and procedures responsible of heath care associated infection $[2,3]$. Endocarditis incurred by Streptococcus agalactiae in adults (nonpregnant females and men) has increased in recent years [4,5], even though the source of infection due to this bacterium and the portal of entry are unclear and not often well documented.

Concerning the possible sexual port of entry in the cause of endocarditis, this mode of transmission should be suspected in a search of clinical history, especially in recurrent endocarditis.

\section{Case Report}

We report on a 49-year old female with recent adjustment or orthodontic appliances one month after which probably due to the breakdown of mucocutaneous barriers she had an oral abscess, apparently treated successfully with amoxicillin/clavulanate. Microbiological culture of the abscess and blood cultures made at another hospital in Milan revealed the presence of $S$. agalactiae. After non-improvement, the patient was admitted one month later to our emergency department with shoulder pain on movement that did not change for 5 days. Physical examination revealed a low grade fever $\left(38^{\circ} \mathrm{C}\right)$. Laboratory testing revealed leukocytosis $(13,540 / \mu \mathrm{l})$, platelet count $(474,000 / \mu \mathrm{l})$, C-reactive protein $(50 \mathrm{mg} / \mathrm{L})$, and D dimer $(286 \mathrm{ng} /$ dl). Empirical therapy with ciprofloxacin was begun after collecting two sets of blood cultures and a urine sample.

During hospitalization in the Infectious Department, she underwent transesophageal and transthoracic echocardiography (Figure 1) that confirmed the presence of endocarditis on the anterior (A2) and posterior (P2-P3) leaflets of the mitral valve and with a free floater mass in the left ventricle having a base of about $1 \mathrm{~cm}$ and a diameter of $2 \mathrm{~cm}$.

The suspected endocarditis and septic peripheral embolization was confirmed. Blood cultures had been negative during admission to our hospital, thus the patient was treated with gentamicin and ampicillin in vain.

According to the modified Duke criteria for infective endocarditis (IE), the patient was taken to the emergency operating room based on a potential embolism.
The native mitral valve was repaired by two triangular resection of A2 and P2-P3. Leaflets reconstruction and anuloplasty with Carpentier Edwards Physio II n.28 (Edwards Lifesciences Corp, Irvine, CA) completed the procedure that was performed through a small right thoracotomy. The resected leaflet and vegetations were sent to the microbiological laboratory for the analysis.

A traditional culture with a 5-day subculture was performed with negative results for bacteria (aerobes and anaerobes) and fungi; a dithiothreitol procedure obtained during surgery was positive for S. agalactiae.

The postoperative course was free from major complications, and the patient was discharged and sent home with instructions to continue antibiotic therapy with ceftriaxone for 4 weeks. However, approximately a month post-surgery, the patient had an increase in C-reactive protein $(70 \mathrm{mg} / \mathrm{L})$ and fever $\left(38^{\circ} \mathrm{C}\right)$, entailing a reassessment of the patient with a possible diagnosis of postsurgical pericarditis.

Further microbiological investigation was performed. Discussion with the patient and her husband, asymptomatic for urogenital discomfort or discharge, revealed that the couple had had oral sex while the husband was under orthodontic treatment a few days before the first emergency room episode. An oral swab determined he was colonized orally by $S$. agalatiae. We hypothesize that the vaginal infection due to $S$. agalactiae pre-surgery and during the dental work was caused by the entrance of the pathogen into the patient's circulation and therefore was responsible for infection of the valve.

IE risk factors have been identified [6], and they include age over 60 years and a diagnosis of diabetes, cancer, chronic renal disease, and

*Corresponding author: Sara Giordana Rimoldi, Laboratory of Clinical Microbiology, Virology and Diagnostics Bioemergenze, ASST Fatebenefratelli Sacco-Polo Universitario, Via G.B. Grassi 74, 20157 Milan, Italy, Tel: +390239042589; Fax: +39-0239042313; E-mail: sara.rimoldi@asst-fbf-sacco.it

Received January 28, 2017; Accepted March 04, 2017; Published March 07 2017

Citation: Rimoldi SG, Vanelli P, Merli S, Monica C, Rubinia R, et al. (2017) An Oral Abscess as the Entrance Leading to Endocarditis: A Sexual Behavior Role. J Pulm Respir Med 7: 397. doi: 10.4172/2161-105X.1000397

Copyright: (c) 2017 Rimoldi SG, et al. This is an open-access article distributed under the terms of the Creative Commons Attribution License, which permits unrestricted use, distribution, and reproduction in any medium, provided the original author and source are credited. 
Citation: Rimoldi SG, Vanelli P, Merli S, Monica C, Rubinia R, et al. (2017) An Oral Abscess as the Entrance Leading to Endocarditis: A Sexual Behavior Role. J Pulm Respir Med 7: 397. doi: 10.4172/2161-105X.1000397

Page 2 of 2

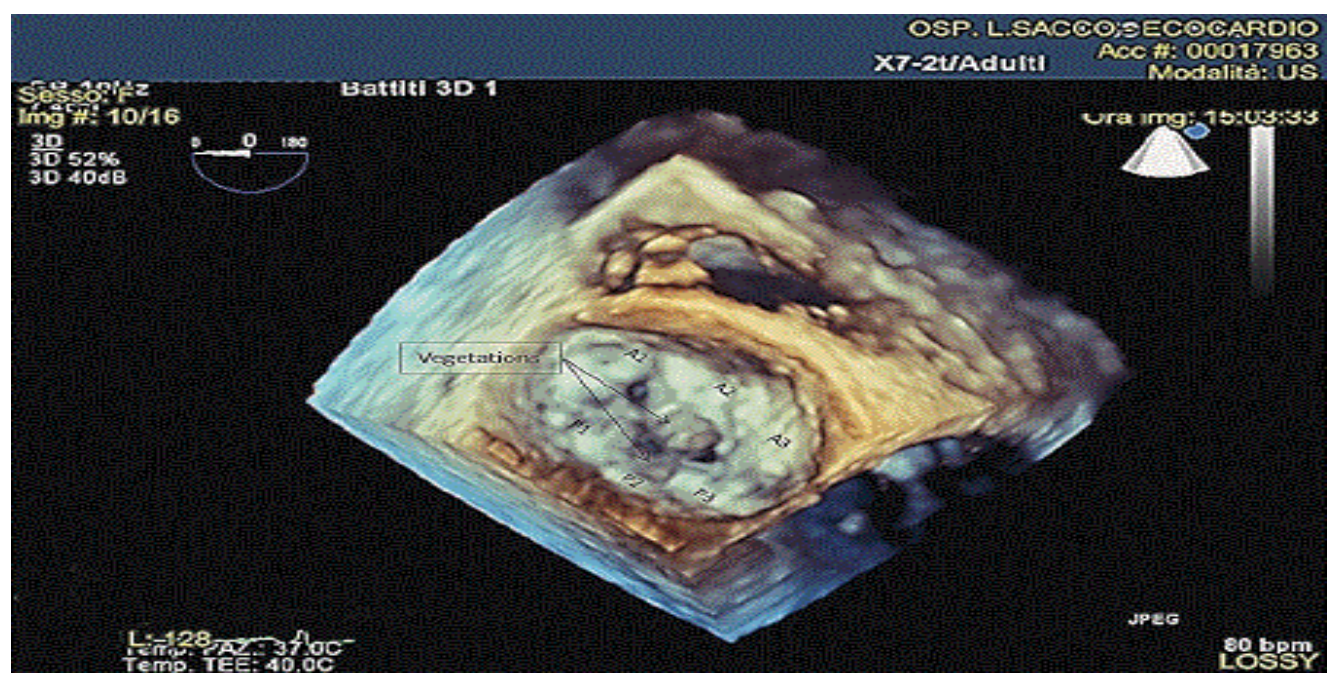

Figure 1: Real-time 3D transoesophageal echocardiography volume rendering of the mitral valve. Two vegetations on the anterior and posterior mitral leaflet (arrows). A1, A2, A3, anterior mitral valve scallops; P1, P2, P3, posterior mitral valve scallops.

neurological vessel disorder; however, in evaluating the medical history, one should consider sexual habits and dental status.

Even though it has been reported [7] that IE can be induced by bacteremia attributable to dental procedures as happened to our patient this is the first time that colonization/vaginal infection of a woman has been suspected of being transferred through oral sex.

\section{Comment}

Although S. agalactiae is responsible for various clinical forms of skin, soft tissue, bone, and urinary tract infections [8] most commonly occurring between mother and neonate more rarely it could be a pathogen involved in endocarditis. Even if, in the past few years, there have been epidemiological changes in the bacterium responsible for the infections [9], S. agalactiae is a rare cause of IE $(1.7 \%$ of all cases) [10]. We believe that the change of risk factors for endocarditis should factor in sexual habits, especially in view of the increased frequency of sexually transmitted bacterial endocarditis. To support our theory, we call attention to 18 papers in the literature reporting that $S$. agalactiae and other pathogens have been suspected of being sexually transmitted. However, these cases have never been investigated as causative of IE. Neisseria gonorrhea has been reported as a cause of endocarditis, especially in young males, although not necessarily reporting urogenital discomfort or urethral discharge [11]. Chlamydia trachomatis, Mycoplasma hominis [12], like Ureaplasma urealyticum and Gardnerella vaginalis, can induce cardiovascular disease, and endocarditis following respiratory tract infection as an entrance door. Transmission of endocarditis through sex has never been documented [13].

The Centers for Disease Control and Prevention has campaigned in an effort to raise awareness among adolescents (aged 13-24) about the sexual behaviors at risk for venereal diseases, infections, and unintended pregnancies due early coitarca.

Therefore, our work should focus on the new population at risk for endocarditis, other than the injecting drug users dying of endocarditis, which have been typical of the past decade; sexual behavior should not be omitted in evaluating a medical history.

\section{Acknowledgment}

We thank Dott Pagani Cristina, Palazzin Alessandro and Di Gregorio Annamaria for their microbiological support.

\section{References}

1. Forestier E, Fraisse T, Roubaud-Baudron C Selton-Suty C, Pagani L (2016) Managing infective endocarditis in the elderly: new issues for an old disease. Clin Interv Aging 11: 1199-1206.

2. Sharma A, Duraisamy S, Jagia P, Gulati GS (2017) Rare iatrogenic cardiovascular embolization following dialysis. Vasc Endovascular Surg 51 33-35.

3. Polewczyk M, Jachec W, Polewczyk AM, Polewczyk A, Czajkowski M, et al. (2016) Leads dislodged into the pulmonary vascular bed in patients with cardiac implantable electronic devices. Adv Interv Cardiol 12.4: 348-354.

4. Abdelghany $M$, Schenfeld L (2014) Group B streptococcal infective endocarditis. J Infect Public Health 7: 237-239.

5. Kuijpers JM, Koolbergen DR, Groenink M, Peels KC, Reichert CL, et al. (2017) Incidence, risk factors, and predictors of infective endocarditis in adult congenital heart disease: focus on the use of prosthetic material. Eur Heart $\mathrm{J}$ : ehw591.

6. Jackson LA, Hilsdom R, Farley MM Harrison LH, Reingold AL, et al. (1995) Risk factors for group B streptococcal disease in adults. Ann Intern Med 123 415-420.

7. Ariyoshi N, Miyamoto K, Bolger DT (2016) Streptococcus agalactiae mura infective endocarditis in a structurally normal heart. $\mathrm{J}$ community Hosp Int Med Perspectives 6: 10.3402/jchimp.v6.31113.

8. Van der Mee-Marquet N, Fourny L, Arnault L, Domelier AS, et al. (2008) Molecular characterization of human colonizing Streptococcus agalactiae strains isolated from throat, skin, anal margin and genital body site. J Clin Microbiol 46: 2906-2911.

9. Murdoch DR, Corey GR, Hoen B Miró JM, Fowler VG Jr, et al. (2009) Clinica presentation, etiology, and outcome of infective endocarditis in the 21 st century: the International Collaboration on Endocarditis-Prospective Cohort Study. Arch Intern Med 169: 463-473.

10. Phares CR, Lynfield R, Farley MM, Mohle-Boetani J, Harrison LH, et al. (2008) Epidemiology of invasive group B streptococcal disease in the United States, 1999-2005. JAMA 299: 2056-2065.

11. Rimoldi SG, De Vecchi E, Pagani C, Zambelli A, Di Gregorio A, et al. (2016) Use of Dithiothreitol to Dislodge bacteria from the biofilm on an aortic valve in the operating theatre: A case of infective Endocarditis caused by Staphylococcus aureus and Proteus mirabilis. Ann Thorac Surg 102: e357-e359.

12. Ramos A, Garcia Pavia P, Orden B, Cobo M, Sánchez-Castilla M, et al. (2014) Gonococcal endocarditis: a case report and view of literature. Infection 42: 425-428.

13. Gagneux-Brunon A, Grattard F, Morel J, Suy F, Fuzellier JF (2015) Mycoplasma hominis: a rare but true cause of infective endocarditis. J Clin Microbiol 53: 3068-3071. 